\title{
THE HIGH-ENERGY, ARCMINUTE-SCALE GALACTIC CENTER GAMMA-RAY SOURCE
}

\author{
M. Chernyakova ${ }^{1}$, D. Malyshev ${ }^{1}$, F. A. Aharonian ${ }^{1,2}$, R. M. Crocker ${ }^{2}$, and D. I. Jones ${ }^{2}$ \\ ${ }^{1}$ Dublin Institute for Advanced Study, Astronomy \& Astrophysics Section, 31 Fitzwilliam Place, Dublin 2, Ireland; masha@cp.dias.ie \\ 2 Max Planck Institut für Kernphysik, Postfach 103980, 69029 Heidelberg, Germany \\ Received 2010 September 13; accepted 2010 October 22; published 2010 December 15
}

\begin{abstract}
Employing data collected during the first 25 months of observations by the Fermi-LAT, we describe and subsequently seek to model the very high energy (>300 MeV) emission from the central few parsecs of our Galaxy. We analyze the morphological, spectral, and temporal characteristics of the central source, 1FGL J1745.6-2900. The data show a clear, statistically significant signal at energies above $10 \mathrm{GeV}$, where the Fermi-LAT has angular resolution comparable to that of HESS at TeV energies. This makes a meaningful joint analysis of the data possible. Our analysis of the Fermi data (alone) does not uncover any statistically significant variability of 1FGL J1745.6-2900 at $\mathrm{GeV}$ energies on the month timescale. Using the combination of Fermi data on 1FGL J1745.6-2900 and HESS data on the coincident, TeV source HESS J1745-290, we show that the spectrum of the central gamma-ray source is inflected with a relatively steep spectral region matching between the flatter spectrum found at both low and high energies. We model the gamma-ray production in the inner $10 \mathrm{pc}$ of the Galaxy and examine cosmic ray (CR) proton propagation scenarios that reproduce the observed spectrum of the central source. We show that a model that instantiates a transition from diffusive propagation of the $\mathrm{CR}$ protons at low energy to almost rectilinear propagation at high energies can explain well the spectral phenomenology. We find considerable degeneracy between different parameter choices which will only be broken with the addition of morphological information that gamma-ray telescopes cannot deliver given current angular resolution limits. We argue that a future analysis performed in combination with higher-resolution radio continuum data holds out the promise of breaking this degeneracy.
\end{abstract}

Key words: cosmic rays - diffusion - Galaxy: center - gamma rays: general

Online-only material: color figures

\section{INTRODUCTION}

Over the past decade-and-a-half since the discovery by EGRET of a very high energy (VHE) gamma-ray source near the Galactic center (GC), there has been intense speculation as to what mechanism(s) are producing the observed emission. The subsequent discovery of $\mathrm{TeV}$ gamma-ray emission from the Sgr A* region by a number of ground-based gamma-ray instruments (Aharonian et al. 2008 and references therein) has generated further theoretical activity. Of general interest and import-given that the GC constitutes the nearest example of a galactic nucleus - is the identity of the sites where (and mechanism(s) by which) particles are accelerated to $\mathrm{TeV}$ energies and beyond in Sgr A* - the dynamical center of our Galaxy.

Despite the fact that the GC TeV gamma-ray source is a point-like object for the HESS array of atmospheric Cherenkov telescopes (Aharonian et al. 2004), the 0.07 point-spread function (PSF) of the instrument and the extremely crowded and complex nature of the region (as evidenced by the complex radio morphology: Law et al. 2008) do not allow the unambiguous identification of the source(s) of gamma-ray emission. With the latest data, however, it is possible to place the center of gravity of the TeV point source within the central $\sim 6^{\prime \prime}$ of the Galaxy (Acero et al. 2010), leaving only a handful of possible sources. These include the central black hole itself, Sgr A* (Aharonian \& Neronov 2005a; Liu et al. 2006a); a plerion discovered within the central few arcseconds of the Galaxy (Wang et al. 2006; Hinton \& Aharonian 2007), a putative "black hole plerion" produced by the wind from Sgr A* (Atoyan \& Dermer 2004), and the diffuse $\leqslant 10$ pc region surrounding Sgr A* (Aharonian \& Neronov 2005b; Ballantyne et al. 2007, 2010; Fryer et al. 2007).

Here we consider further insights now possible in light of the Fermi-LAT observations of the GC region. In particular, since the PSF of Fermi above $\sim 10 \mathrm{GeV}$ is similar to that of HESS, it is now possible to explore relativistic particles localized in the $\mathrm{GC}$ region over quite a broad energy interval.

The results of the first 11 months of Fermi observations of the GC were presented by Cohen-Tanugi on behalf of the Fermi-LAT Collaboration during the 2009 Fermi Symposium (preliminary results based on first 6 months of observations are given by Cohen-Tanugi et al. 2009). In this work, the authors argued that the Fermi source 1FGL J1745.6-2900 and the HESS source J1745-290 are spatially coincident. They also derived the energy spectrum of the Fermi source up to $100 \mathrm{GeV}$ and concluded that to match the HESS spectrum either a high-energy down-break or cutoff is required. A discussion of the first 11 months of Fermi observations of GC in relation to the search of dark matter can be found in Vitale et al. (2009).

In this work, we analyze the first 25 months of Fermi-LAT data. In addition to the central $\mathrm{GeV}$ and other reported sources, our analysis reveals four new sources of $\mathrm{GeV}$ gamma-rays located in this region. With the spectral information from both Fermi and HESS in hand, we model the production of gammarays from the inner $\mathrm{GC}$ due to hadronic interactions of protons accelerated within the central black hole and diffusing into the surrounding interstellar medium.

In Section 2, we describe the reduction and analysis of the Fermi data. We present the details of our model in Section 3. In Section 4, we discuss the implications of the obtained results and summarize the main conclusions in Section 5.

\section{DATA ANALYSIS AND RESULTS}

The large area telescope (LAT) on board the Fermi satellite is a pair-conversion gamma-ray detector operating between $20 \mathrm{MeV}$ 

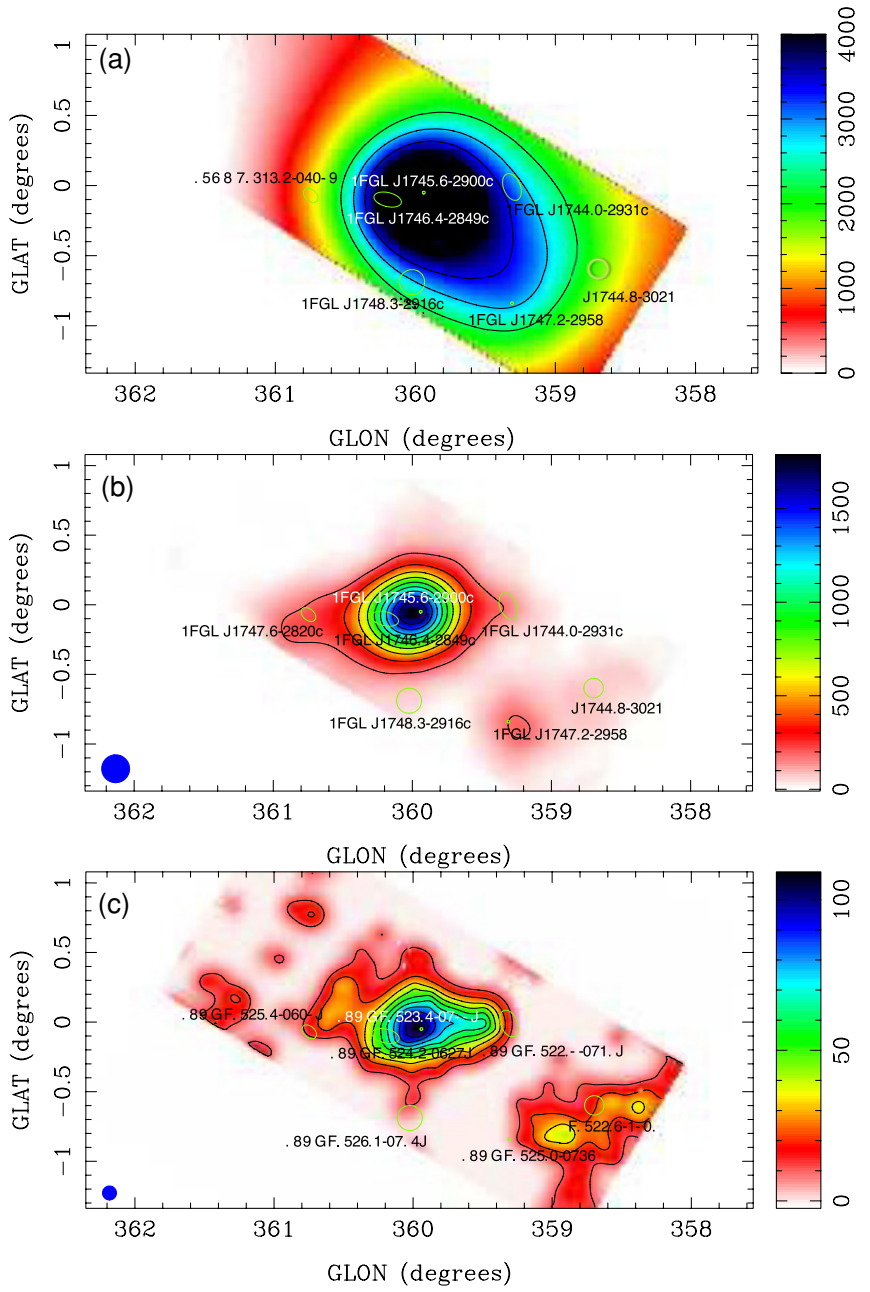

Figure 1. Test statistic maps of the central $1.5 \times 3.5$ of the Galaxy as seen by Fermi in the energy ranges of (a) $300 \mathrm{MeV}-3 \mathrm{GeV}$, (b) $3 \mathrm{GeV}-30 \mathrm{GeV}$, and (c) $30 \mathrm{GeV}-300 \mathrm{GeV}$. Positions of the sources are marked with ellipses according to the one year Fermi catalog (1FGL). The position of the new source $\mathrm{J} 1744.8-3021$ is given in Table 1 and is denoted with a circle in the images. Note that the color scheme has different maximum values in all cases varying from 4000 in panel (a) to 140 in (c). Source significance can be approximately estimated as the square root of TS. The black contours are set in steps of $10 \%$ at levels of $10 \%-90 \%$ of the peak emission, in steps of $10 \%$ for (b) and (c) and from $40 \%$ to $90 \%$ for (a). In the lower left-hand corner of images (b) and (c) we show Fermi at $3 \mathrm{GeV}(0.3)$ and $30 \mathrm{GeV}(0.1)$. The PSF at $300 \mathrm{MeV}\left(2^{\circ}\right)$ is too large to show on panel (a), but note that likelihood analysis allows us to resolve sources on size scales much smaller than PSF.

and $300 \mathrm{GeV}$. The LAT has a wide field of view of $\sim 2.4 \mathrm{sr}$ at $1 \mathrm{GeV}$ and observes the entire sky every two orbits $(\sim 3 \mathrm{hr}$ for a Fermi orbit at an altitude of $\sim 565 \mathrm{~km}$; full details of the instrumentation are given in Atwood et al. 2009). The data used for our analysis are based on the first 25 months of observations (2008 August 4-2010 August 18).

Data analysis was performed using the LAT Science Tools package with the P6_V3 post-launch instrument response function (Rando et al. 2009). Standard event selection for source analysis resulting in the strongest background-rejection power (diffuse event class) was applied. In addition, photons coming from zenith angles larger than $105^{\circ}$ were rejected to reduce the background from gamma rays produced in the atmosphere of the Earth. The analysis was further restricted to the energy range above $100 \mathrm{MeV}$ since below this energy the telescope's effective area becomes very small and the residual uncertainty in the instrumental response significant.

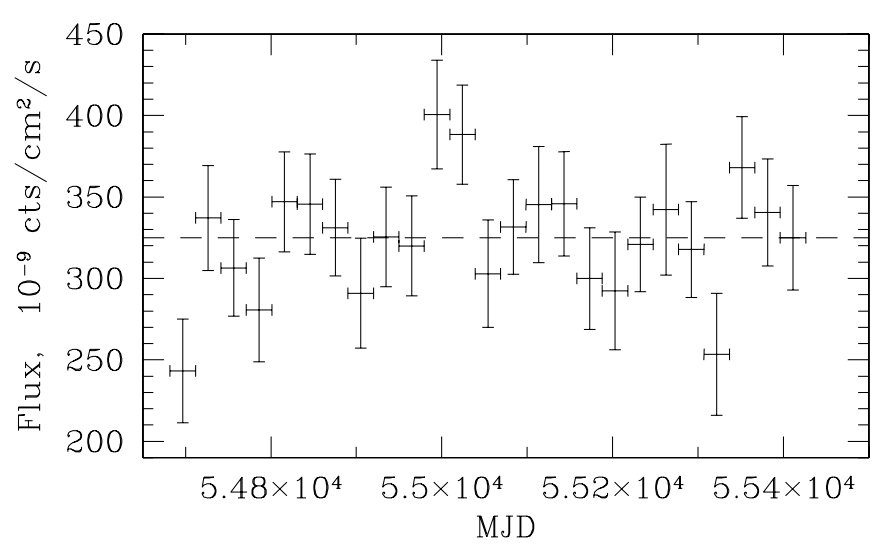

Figure 2. Light curve of 1FGL J1745.6-2900 in the $300 \mathrm{MeV}-100 \mathrm{GeV}$ energy range. The average flux is shown with a dashed line.

Table 1

Coordinates and TS of the New Sources Discovered During the Analysis

\begin{tabular}{ccc}
\hline \hline $\begin{array}{c}\text { R.A. } \\
(\mathrm{J} 2000.0)\end{array}$ & $\begin{array}{c}\text { Decl. } \\
(\mathrm{J} 2000.0)\end{array}$ & TS \\
\hline 264.906 & -28.555 & 331 \\
266.210 & -30.360 & 424 \\
270.060 & -30.091 & 189 \\
270.697 & -30.626 & 192 \\
\hline
\end{tabular}

In order to take into account the broad PSF at low $(\sim 100 \mathrm{MeV})$ energies, we constructed a sequence of test statistic (TS) images of the $10^{\circ} \times 10^{\circ}$ region around Sgr $\mathrm{A}^{*}$. In producing the TS images, we used the gttsmap tool with a tolerance parameter of ftol $=10^{-5}$ and a bin size in each map of 0.1 . After subtracting the 19 known sources from the one year Fermi catalog (1FGL) which happen to be within the selected region, we found four new sources in the residual images, the details of which are listed in Table 1. One of these sources (indicated as J1744.8-3021) - shown in Figure 1-lies within the $1.5 \times 3^{\circ}$ area around the GC. This source coincides spatially with known HESS source J1745-303 and EGRET source 3EGJ1744-3011.

In order to construct a light curve for 1FGL J1745.6-2900, we used a spectral method by selecting data in the $300 \mathrm{MeV}-$ $100 \mathrm{GeV}$ energy range and fitting all known sources, selected as above, with a single power-law model. Afterward, we split the whole time interval into 25 equal time bins and fit source spectra by fixing their slopes to the best-fit value obtained over the entire time period, leaving the source normalization as a free parameter. The normalization of the Galactic and extra-galactic background was also left as a free parameter in our fitting. The resulting light curve, shown in Figure 2, is relatively stable and does not exhibit any statistically significant variation. The average flux is equal to $(324.9 \pm 7.05) \times 10^{-9}$ counts $\mathrm{cm}^{-2} \mathrm{~s}^{-1}$, with a reduced $\chi^{2}=1.1$ for 24 degrees of freedom. Spectral fitting was performed within $100 \mathrm{MeV}-300 \mathrm{GeV}$ energy range with the gtlike tool and the resulting spectrum is shown in Figure 3.

The gamma-ray data can be described with a single power law (dashed line in Figure 3) with best-fit parameters $\Gamma=$ $2.43 \pm 0.03$ and a flux normalization of $F=(2.81 \pm 0.3) \times$ $10^{-10} \mathrm{erg} \mathrm{cm}^{-2} \mathrm{~s}^{-1}$ at $100 \mathrm{MeV}$, but the fit for this is poor: $\chi^{2} /$ dof $=2.84$ for 10 degrees of freedom (dof). A broken power law (solid line in Figure 3) with break energy $E_{\mathrm{br}}=$ $2.0_{-1.0}^{+0.8} \mathrm{GeV}$ gives a much better fit: $\chi^{2} /$ dof $=0.81$ for 9 dof. The resulting slopes before and after break energy are equal to $\Gamma_{1}=2.20 \pm 0.04\left(E<E_{\mathrm{br}}\right)$ and $\Gamma_{2}=2.68 \pm 0.05\left(E>E_{\mathrm{br}}\right)$, 


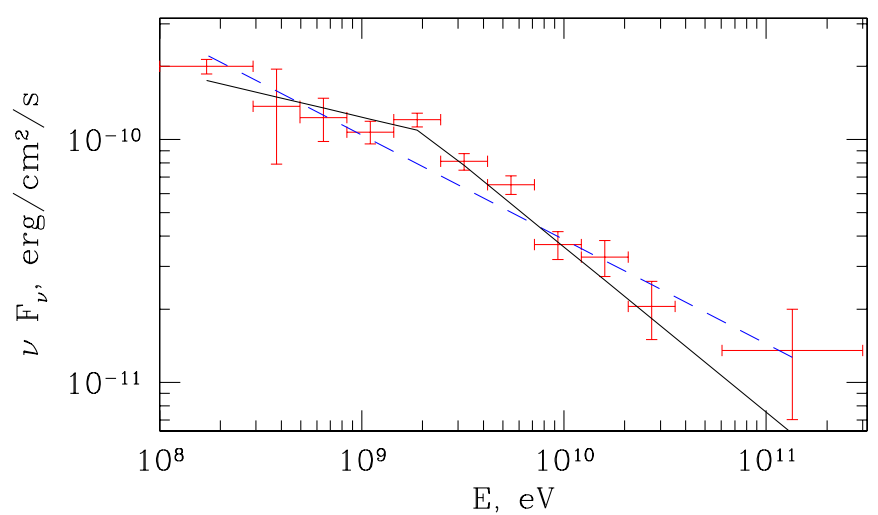

Figure 3. Spectrum of the source 1FGL J1745.6-2900 in $100 \mathrm{MeV}-300 \mathrm{GeV}$ energy range. Dashed and solid curves correspondingly show the best-fit single $\left(\chi^{2} /\right.$ dof $\left.=2.84\right)$ and broken $\left(\chi^{2} /\right.$ dof $\left.=0.81\right)$ power-law models.

(A color version of this figure is available in the online journal.)

respectively, and the flux normalization at $2 \mathrm{GeV}$ is equal to $F=(1.08 \pm 0.06) \times 10^{-10} \mathrm{erg} \mathrm{cm}^{-2} \mathrm{~s}^{-1}$. The errors given above are statistical errors only and represent the $1 \sigma$ deviation. Thus, the slope of the Fermi spectrum above several $\mathrm{GeV}$ is significantly steeper than the spectrum reported by the HESS Collaboration at TeV energies $\left(F_{\text {HESS }} \sim E^{-2.1}\right.$; Aharonian et al. 2009). Note that at low energies, Fermi has a very broad PSF, rapidly moving from $4^{\circ}$ at $100 \mathrm{MeV}$ to $2^{\circ}$ at $300 \mathrm{MeV}$. Thus, taking into account the possible source confusion in the region, one should treat the first datum in the spectrum (100-300 MeV) with caution.

\section{MODELING}

As proposed in Aharonian \& Neronov (2005b), a significant fraction of the protons accelerated near the black hole may enter the surrounding gaseous environment and initiate VHE gammaray emission through neutral pion production and subsequent decay. The efficiency of the process and the energy spectrum of the resulting gamma-rays depend not only on the protons' injection rate and the ambient gas density, but also on the speed of proton transport into the surrounding medium (Aharonian \& Neronov 2005b; Ballantyne et al. 2007, 2010). To explain the gamma-ray spectrum reported by the HESS Collaboration, Aharonian \& Neronov (2005b) assumed that relativistic protons with a power-law spectrum possessing a spectral index of $\Gamma \sim 2$ are injected into the dense gaseous environment surrounding the central black hole. The diffusion coefficient, $D$, was assumed to have a power-law dependence on energy of the form $D(E)=$ $10^{28}(E / 1 \mathrm{GeV})^{\beta} \kappa \mathrm{cm}^{2} \mathrm{~s}^{-1}$. For the cosmic ray (CR) diffusion in the Galactic disk $\kappa \sim 1$ and $\beta \sim 1$, but of course the diffusion coefficient in the GC could be quite different. Aharonian \& Neronov (2005b) assumed that the diffusion parameter $\beta$ was in the range of $0.5-1$. In the modeling of Ballantyne et al. (2007, 2010), however, propagation is treated using a ray-tracing technique and in order to reproduce the reported energy distribution of $\mathrm{TeV}$ gamma-rays, the spectrum of protons should be hard with a spectral index $\sim 0.75$. Such an exceptionally hard injection spectrum of protons implies a very strong energy dependence for protons which, within the formalism of diffusion, would require a diffusion coefficient with $\beta \sim 1.5$. In our modeling, we have assumed a range of values for $\beta$ and discuss the effects on the resulting gamma-ray spectrum below.
Given that the VHE emission detected by HESS and Fermi can be localized to within the central several arcminutes then, for a GC distance of $d \sim 8 \mathrm{kpc}$, the linear size of the production region of VHE gamma-rays can be as large as $R \sim 10$ pc. It should be noted that, in this case, we do not expect variability from the GC sources to be present on time scales below $R / c \sim 10$ years, in agreement with the HESS and Fermi data. This justifies the combination of the HESS and Fermi data for spectral analysis, despite the fact that HESS and Fermi observations were not simultaneous (HESS observed GC in 2004-2006, and Fermi observations discussed here were performed from 2008 to 2010).

Continuum X-ray and radio observations of the central $10 \mathrm{pc}$ region of the Galaxy show a complex environment with many unique structures (Law et al. 2008). It is thought that molecular matter is distributed around the GC in a torus, the so-called circum-nuclear disk, of inner radius $\sim 1$ pc (see, e.g., Ballantyne et al. 2007; Fryer et al. 2007). There are, however, significant uncertainties in characterizing the amount and distribution of gas in this complex environment. So as not to obscure the essential physics, we therefore limit ourselves to spherical symmetry in this paper. In particular, inspired by the parameters of what is thought to be the real matter distribution, we take it that the supermassive black hole in the center of our Galaxy is surrounded by a shell of a dense matter with a density normalized to $n_{\mathrm{H}}=1000 \mathrm{~cm}^{-3}$ at $1 \mathrm{pc}$ radius, with either constant or $1 / r^{2}$ radial dependence. The inner and outer radii of this shell are parameters in our model. Another relevant parameter is the time evolution of the proton injection; although one can treat this as a quasi-stationary process, the proton injection can, in fact, be dominated by one (or several) past flare(s) from Sgr $\mathrm{A}^{*}$. In this context, we mention the morphological interpretation of the diffuse gamma-ray emission observed by HESS from the central $200 \mathrm{pc}$ region of the GC, which relates the positive detections of gamma-rays from giant molecular clouds in the GC to a putative "proton" flare that occurred in Sgr A* in the past $\sim 10,000$ years ago (Aharonian et al. 2006). The detection of reflected X-ray emission from the Sgr B2 cloud is another, more direct piece of evidence for flaring activity of Sgr A* a few hundred years ago (Sunyaev et al. 1993; Koyama et al. 1996, 2008; Revnivtsev et al. 2004; Terrier et al. 2010). Given these pieces of evidence, we have, in our model, explored how either a single (or multiple) flare(s) affect the resultant gamma-ray emission in the GC region.

In the standard diffusion approximation, the propagation of particles is described by the diffusion equation (Ginzburg \& Syrovatskii 1964) which, in the spherically symmetric case, reduces to the form:

$$
\frac{\partial n}{\partial t}=\frac{D}{r^{2}} \frac{\partial}{\partial r} r^{2} \frac{\partial n}{\partial r}+\frac{\partial}{\partial E}(\mathrm{bn})+\mathrm{Q},
$$

where $n(r, t, E)$ is the space density of relativistic particles with energy $E$, at instant $t$ being a distance $r$ from the source; $b(E)=-d E / d t$ is the continuous energy loss rate; $Q(E, t)$ is the injection rate; and $D(E)$ is the (energy-dependent) diffusion coefficient. We have assumed here, for simplicity, that $D$ is independent of $r$ and has a power-law dependence on energy as stated above. The solution of Equation (1) can be written as (Syrovatskii 1959)

$$
n(E, r, t)=\int_{0}^{t} P(E, r, t-x) Q(E, x) d x,
$$




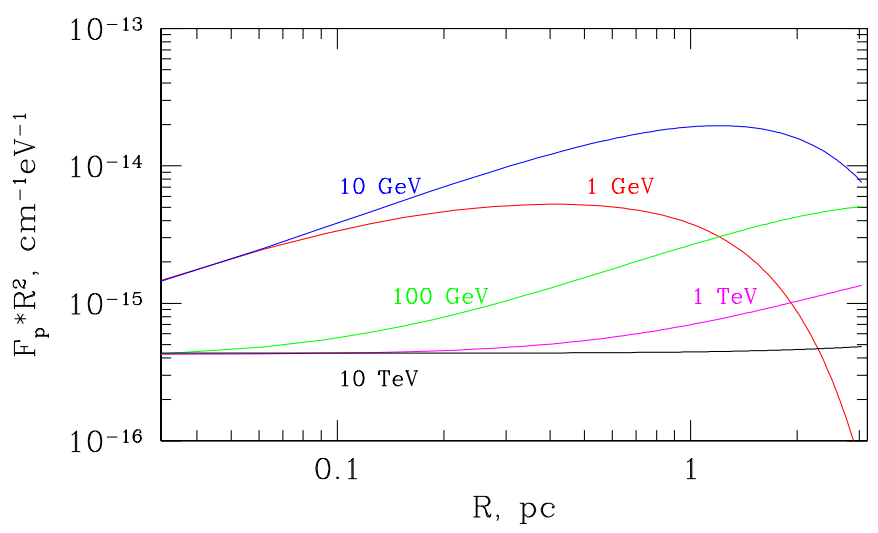

Figure 4. Proton distribution calculated after 300 years of continuous injection into the interstellar medium within a region of radius $R=3 \mathrm{pc}$ (see the text for details). The fluxes of protons (as a function of radius) at different energies are shown as marked. For ease of comparison each energy was multiplied by a factor of 900 (for $10 \mathrm{GeV}), 8000(100 \mathrm{GeV}), 5 \times 10^{6}(1 \mathrm{TeV})$, and $7 \times 10^{8}$ $(10 \mathrm{TeV})$. The flux at each energy has been multiplied by $R^{2}$, so that rectilinear propagation corresponds to a horizontal line.

(A color version of this figure is available in the online journal.)

where the propagator, $P(E, r, t)$, is defined as

$$
P(E, r, t)=\frac{1}{[4 \pi \lambda(E, t)]^{3 / 2}} \exp \left[-\frac{r^{2}}{4 \lambda(E, t)}\right],
$$

and

$$
\lambda(E, t)=-\int_{E_{g}(t)}^{E} d x \frac{D(x)}{b(x)} .
$$

In Equation (4), $E_{g}$ is the energy that a cooled particle possesses at time $t$, if its initial energy was $E$.

Formally, the diffusion equation does not contain information on how fast a particle may propagate. Since Equation (1) does not prevent artificial "superluminal motion" $\left(v_{0}=2 D(E) / r \gtrsim c\right)$, we follow the phenomenological approach proposed by Aloisio et al. (2009) who introduced a propagator, $P(E, r, t)$ of the form:

$$
\begin{aligned}
P(E, r, t)= & \frac{\theta(1-\xi)}{4 \pi(c t)^{3}} \frac{1}{\left(1-\xi^{2}\right)^{2}} \frac{\alpha(E, \xi)}{K_{1}[\alpha(E, \xi)]} \\
& \times \exp \left[-\frac{\alpha(E, \xi)}{\sqrt{1-\xi^{2}}}\right]
\end{aligned}
$$

where $\theta(x)$ is the Heaviside step function, $\xi(t)=r / c t, K_{1}(x)$ is the modified Bessel function of the second kind, and $\alpha(E, t)$ is defined as

$$
\alpha(E, t) \equiv \frac{c^{2} t^{2}}{2 \lambda(E, t)},
$$

where $E_{c}$ is the energy at which Equation (1) allows diffusion with the speed of light:

$$
E_{c}=\left(\frac{c R}{2 D_{0}}\right)^{1 / \beta} \mathrm{GeV} .
$$

In the low-energy regime (i.e., $E \ll E_{c}$ ), the propagator given by Equation (5) reproduces the standard treatment of diffusion, while in the high-energy regime $\left(E \gtrsim E_{c}\right)$ it describes particles that move in a rectilinear fashion.

Due to the energy dependence of the diffusion coefficient, proton propagation will be quite different at low energies as

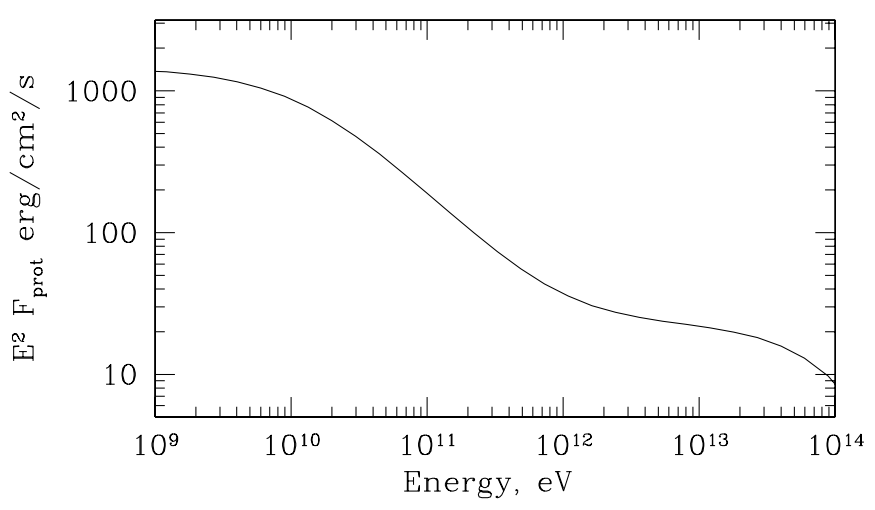

Figure 5. Spectrum of the protons integrated over the $3 \mathrm{pc}$ of gamma-ray production region. Photons produced by the interaction of relativistic protons with such an energy distribution fit both Fermi and HESS data well.

compared to high energies: we have explored how this plays out in the GC environment. The result presented in Figure 4 shows the change of the radial distribution of protons as a function of energy. We have determined the proton distribution after 300 years of continuous injection into the interstellar medium of density $n_{\mathrm{H}}=10^{3} \mathrm{~cm}^{-3}$ within a region of radius $R=3$ pc. The initial spectrum of protons was assumed to have a power-law distribution with an exponential cutoff, $Q(E) \propto E^{-2} \exp \left(-E / E_{\text {cut }}\right)$, where, unless otherwise noted, we adopt $E_{\text {cut }}=100 \mathrm{TeV}$ as informed by the observed highenergy cutoff in the HESS photon spectrum (note that the exact cutoff energy is phenomenologically constrained to lie in the approximate range 50-100 TeV: this is explored in Figure 8).

It can be clearly seen from Figure 4 that, while at $10 \mathrm{TeV}$ the particles pass through the region in an almost rectilinear fashion, at lower energies, protons propagate diffusively. An explanation for the lack of low-energy protons at high radii can be found in the fact that these particles have too low an effective diffusion velocity to travel that far within the given time frame.

The spectrum of the protons integrated over the gamma-ray production region is shown in Figure 5. Photons produced by relativistic protons with such an energy distribution fit both the Fermi and HESS data well. At low $(\mathrm{GeV})$ energies, the diffusion radius is smaller than the region so that protons are accumulated within the inner region and, given the almost energy-independent $p p$ cross-section, mirror the spectrum of the injected protons. On the other hand, at $\mathrm{TeV}$ energies, protons begin to propagate in a rectilinear mode and will again have the form of the injected spectrum, albeit at a lower flux level. Protons with an intermediate energy have a much steeper, diffusionprocessed spectrum representing the transition between the two extremes. The spectral shape of the highest energy gammarays is not affected by propagation effects, since they move rectilinearly. Finally, as stated above, in order to match the spectrum at highest energies reported by HESS (Aharonian et al. 2009), we have assumed an exponential cutoff in the proton spectrum and fixed its position at $100 \mathrm{TeV}$.

Below, we fit parameters which represent the particle injection spectrum, the propagation of the injected protons, and the geometry of the interstellar medium. It is instructive to systematically examine the influence of these model parameters on the resulting spectrum. To do this, we begin with $10^{4}$ years of injection of relativistic protons with a spectrum of the form $Q(E) \propto E^{-1.9} \exp (-E / 100 \mathrm{TeV})$ into a region of $3 \mathrm{pc}$ radius filled by an interstellar gas of constant density, $n_{\mathrm{H}}=10^{3} \mathrm{~cm}^{-3}$ at an injection rate of $Q_{0}=3.9 \times 10^{39} \mathrm{erg} \mathrm{s}^{-1}$. The diffusion 


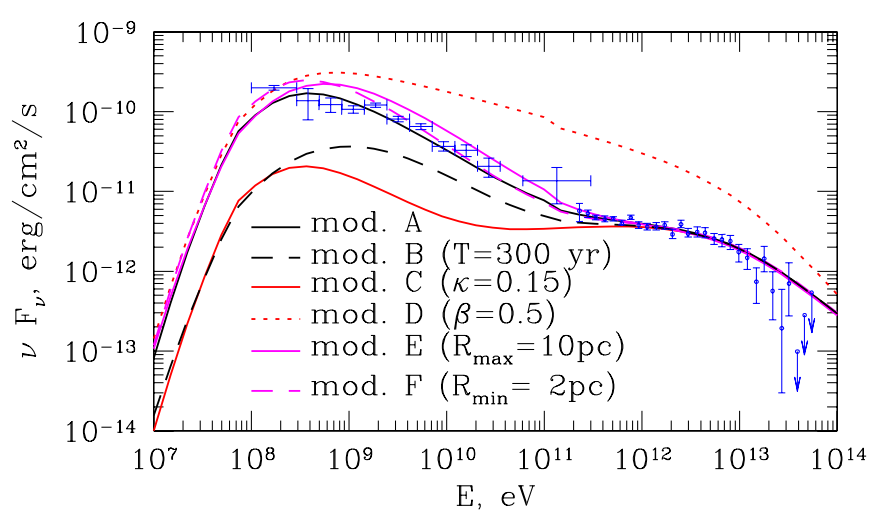

Figure 6. Spectral energy distribution of gamma-rays expected from a region filled with relativistic and non-relativistic protons within different assumptions concerning the injection, diffusion, and the region geometry (see the text for a discussion of parameters for each specific model). The data points have been derived from the Fermi and HESS data.

(A color version of this figure is available in the online journal.)

parameters were chosen as $\beta=0.9, \kappa=0.015$ in order to reproduce the combined Fermi and HESS spectrum. The photon spectrum resulting from this parameter set (model A) is shown in Figure 6. The other curves in Figure 6 illustrate the effect of change of a single parameter (while all other parameters are fixed to the values used in model A).

Model B in Figure 6 corresponds to the case of a source active for only 300 years. This change does not affect highenergy particles traveling rectilinearly, since their escape time, $t_{\text {esc }}=R / c \sim 10$ years, is much shorter than the injection time. These particles fully fill the region and so their density is the same as in the case of model A. The diffusion time at low energies $\left(E<E_{c} \sim 1 \mathrm{TeV}\right.$ in this case), however, is much longer (namely $t_{\text {diff }}=R^{2} / 2 D \sim 9000$ years at $1 \mathrm{GeV}$ ). Thus, 300 years will not be a long enough interval for low-energy particles to travel to the outer regions of the shell, and hence the total spectrum is harder than model A. So, for this parameter set, while the radiation does not differ from our fiducial case at high energies, at lower energies there are necessarily fewer gamma-rays.

Models $\mathrm{C}$ and $\mathrm{D}$ in Figure 6 show what occurs when the diffusion parameters $\kappa$ and $\beta$, respectively, are changed. If one increases the diffusion coefficient, $\kappa$, by a factor of 10 then the diffusion time of the low-energy particles is decreased by the same factor, leading to a corresponding reduction in the intensity of the gamma-ray emission. If one changes the energy dependence of the diffusion by decreasing $\beta$ by a factor of 2 (as in model D), then the transition of the particle's propagation from diffusion to rectilinear propagation occurs at much higher energies $\left(E_{c}\right.$ has $1 / \beta$ dependence-cf. Equation (7)—reaching $\mathrm{PeV}$ energies in this case). Thus, the gamma-ray emission increases at all energies and the spectral form changes reflecting a larger influence of high-energy particles.

Models $\mathrm{E}$ and $\mathrm{F}$ in Figure 6 show that the photon spectrum responds to changes in the geometry of the region. Model E in Figure 6 corresponds to a shell geometry with an outer radius of $R=10 \mathrm{pc}$ (as compared to the $3 \mathrm{pc}$ radius considered in the other models). With this particular geometry, the overall normalization of the resulting spectrum increases due to an increase in size of the gamma-ray production region (a factor of 0.25 was applied to this spectrum for easier comparison to the other models shown in Figure 6). Additionally, the "bump" at lower energies becomes wider, since the energy at

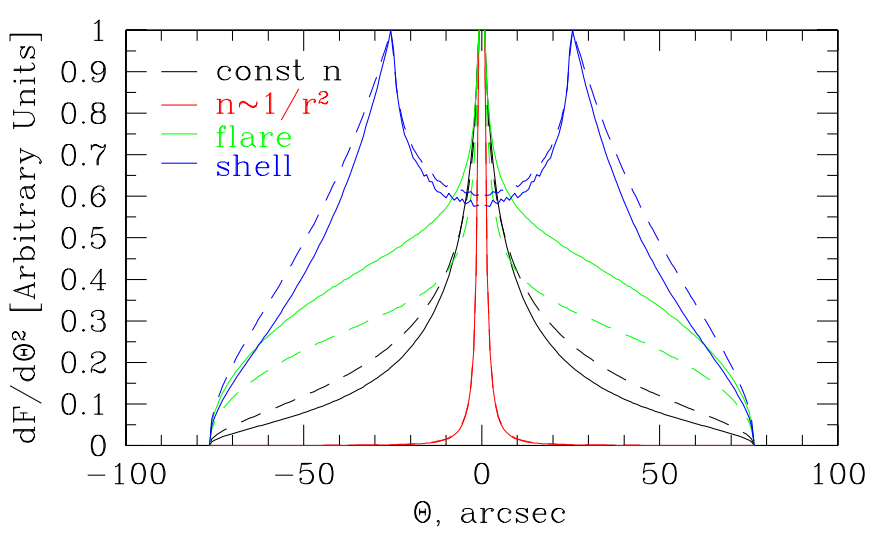

Figure 7. Brightness profile of the GC in $100 \mathrm{MeV}-1 \mathrm{GeV}$ (solid lines) and $1 \mathrm{GeV}-10 \mathrm{GeV}$ (dashed lines) energy ranges. See Table 2 for the description of the models. In this figure, an angular distance of $25^{\prime \prime}$ corresponds to a linear size of $1 \mathrm{pc}$.

(A color version of this figure is available in the online journal.)

which particles transition from the diffusive to the rectilinear propagation regimes increases as $R^{1 / \beta}$, and thus the larger the radius, the larger the number of "intermediate," GeV energy particles that can be accumulated within the region.

The effect of the shell volume is clearly seen in the case when the value of the inner radius of the disk is changed. Model F in Figure 6 represents a shell with inner radius $R_{\min }=2 \mathrm{pc}$ and outer radius $R_{\max }=3 \mathrm{pc}$. In this case, the overall normalization of the resulting spectrum decreases (in Figure 6 a factor of 3.3 has been applied to this spectrum in order to more easily aid the comparison to other models), but the low-energy bump is still wider than in the case of a shell with no hole (i.e., an oblate spheroid). This is due to the fact that removing the inner part of the shell mostly diminishes the soft photon emission, effectively increasing the relative number of more energetic ones. Finally, it should be noted that the sharp drop of gamma-ray spectra below $1 \mathrm{GeV}$ is the result of the kinematics of pion production at $p-p$ interactions and, therefore, does not depend on model parameters.

The radial distribution of photons is also highly dependent on model parameters (cf. Ballantyne et al. 2007; Fryer et al. 2007). Figure 7 shows the brightness profile of the inner 3 pc after $10^{4}$ years of constant proton injection at energies between $100 \mathrm{MeV}-1 \mathrm{GeV}$ (solid lines) and $1 \mathrm{GeV}-10 \mathrm{GeV}$ (dashed lines). For all models the constant source was active for $10^{4}$ years. The initial spectrum of protons is assumed to be a power law with an exponential cutoff, $Q(E) \propto$ $E^{-2} \exp (-E / 100 \mathrm{TeV})$. The median density at $R=1 \mathrm{pc}$ was assumed to be equal to $n_{\mathrm{H}}=10^{3} \mathrm{~cm}^{-3}$. At higher energies, particles pass through the region almost rectilinearly and the region will appear as a point-like source of gamma-rays to Fermi. As labeled in Figure 7, different curves correspond to different model parameter sets in terms of the ambient matter distribution and particle injection. For all models in Figure 7, parameters given in Table 2 were chosen so that the resulting integrated emission accurately reproduced the Fermi and HESS data, and the resulting profiles were normalized to the maximal value in order to aid comparison.

In Figure 7, black lines represent the case of constant density and exhibit a relatively broader profile at higher energies (dashed black line). If instead, the density decreases according to $n_{\mathrm{H}} \propto 1 / r^{2}$, the resulting profiles are thinner (red solid and dashed lines which almost coincide on the figure). The profile is more centrally peaked in the $1 / r^{2}$ case, since the matter is 
Table 2

Parameters of Different Models used for Figure 7

\begin{tabular}{lcllccc}
\hline \hline Model & $n_{\mathrm{H}}$ & $\kappa$ & $\beta$ & $\begin{array}{c}Q_{0} \\
\left(10^{39} \mathrm{erg} \mathrm{s}^{-1}\right)\end{array}$ & $\begin{array}{c}R_{\min } \\
(\mathrm{pc})\end{array}$ & $\begin{array}{c}Q_{f} \\
\left(10^{39} \mathrm{erg} \mathrm{s}^{-1}\right)\end{array}$ \\
\hline Const & Const & 0.02 & 0.95 & 6 & 0 & 0 \\
$1 / r^{2}$ & $1 / r^{2}$ & 0.002 & 0.75 & 0.1 & 0 & 0 \\
Flare & Const & 0.7 & 0.5 & 8 & 0 & 8000 \\
Shell & Const & 0.02 & 0.95 & 8 & 1 & 0 \\
\hline
\end{tabular}

Notes. The medium density at $R=1 \mathrm{pc}$ was assumed to be equal to $n_{\mathrm{H}}=10^{3} \mathrm{~cm}^{-3} \cdot Q_{0}$ and $Q_{f}$ correspond to the injection rates of the constant source and a flare correspondingly.

more concentrated toward the center and so the photon flux will mostly originate from this region. The green lines show the profile created if, in addition to a constant source, there was also a flare which occurred 300 years ago (the injection rate in Table 2 corresponds to a flare of length 10 years). In order to match observational data in this case, a larger diffusion coefficient had to be assumed, which inevitability leads to a larger diffusion radius and-correspondingly - to a wider profile. Finally, in Figure 7 , we show the brightness profiles corresponding to a shell geometry (blue lines). In this case, the profile has a maximum at the radius of the inner shell.

\section{DISCUSSION}

The spectral properties of the VHE emission from the GC differ considerably from that at lower energies: while the GC is known to be variable at X-rays and near-IR wavelengths, no variability has yet been detected either by HESS, or by Fermi. This seeming duality has a natural explanation if the X-rays and near-IR emission is generated very close to the central black hole, while the gamma-ray emission originates from a much larger region and is emitted during the diffusion of the relativistic protons through the interstellar medium surrounding the central black hole. Liu et al. (2006a, 2006b) showed that stochastic acceleration of electrons interacting resonantly with a turbulent magnetic field within a small $(\sim 20$ Schwarzschild radii of Sgr $A^{*}$ ) accretion torus is a plausible production mechanism for much of the Sgr A* millimeter and shorter wavelength emission, as well as near-IR and X-ray flares. Severe synchrotron and synchrotron self-Comptonized losses lead to the immediate reradiation of the electrons' energy. Liu et al. (2006a) also showed that protons are plausibly accelerated in the same region as well, but are able to escape and will lose their energy at much larger distances due to their interaction with the interstellar medium. The impact of such protons on the emission of the Sgr A* itself is discussed by Aharonian \& Neronov (2005a) and depends strongly on the model parameters. In general, VHE emission only reflects (with a delay) major flares originating from the central source.

As remarked above, one interpretation of the distribution of the diffuse, $\mathrm{TeV}$ gamma-ray emission relative to the molecular clouds in the central $\sim 200 \mathrm{pc}$ of our Galaxy is that a central CR proton source flared about $10^{4}$ years ago (Aharonian et al. 2006). In the previous section, we showed that our model is able to reproduce the spectral data if we assume a constant injection of relativistic protons for $10^{4}$ years (see Figure 6). This length of time with constant injection is longer than the diffusion time implied by our parameters set and thus the photon energy spectrum obtained is effectively in a steady state. Our model is able to self-consistently explain different spectral indices at $\mathrm{GeV}$ and $\mathrm{TeV}$ energies by the different effective velocities of the protons. High-energy protons producing $\mathrm{TeV}$ photons escape quasi-rectilinearly and suffer no spectral deformation. Similarly, the low-energy, fully trapped protons are also undistorted given the near energy independence of the $p p$ cross-section (above threshold). In contrast to these cases, particles with intermediate energies are not fully trapped and their spectrum becomes diffusively steepened providing the transition between the two limiting cases.

We would like to stress that the $\mathrm{TeV}$ protons in our model are quite young and left the source about $t \sim R / c \sim 10(R / 3 \mathrm{pc})$ years ago, while the $\mathrm{GeV}$ protons are, necessarily, much older. This allows variability of the source in the TeV energy range on $\sim 10$ year timescales (see also Ballantyne et al. 2010). It also strongly indicates that these protons are accelerated by the central black hole, since we do not currently observe any other source that can pump the required $10^{39} \mathrm{erg} \mathrm{s}^{-1}$ into highenergy protons required to sustain the observed $\sim \mathrm{TeV}$ emission. The required power can be lowered somewhat by changing the density profile (cf. Table 2), the assumption of a more dense ISM, or more effective confinement of protons (Aharonian $\&$ Neronov 2005b). The parameter space for the latter case, however, is rather restricted due to the fact that after the Fermi observations not only the $\mathrm{TeV}$, but also the $\mathrm{GeV}$ observations need to be explained.

Observations of reflected X-ray radiation from the Sagittarius B2 (Sgr B2) cloud located at a distance of $\sim 100 \mathrm{pc}$ from Sgr $A^{*}$ suggest that a few hundred years ago there was an increase of the X-ray luminosity of $\mathrm{Sgr} \mathrm{A}^{*}$ by a factor of 1000 (from $L \sim 10^{36} \mathrm{erg} \mathrm{s}^{-1}$ to $L \sim 10^{39} \mathrm{erg} \mathrm{s}^{-1}$ Sunyaev et al. 1993; Koyama et al. 1996, 2008; Revnivtsev et al. 2004; Terrier et al. 2010). Recent monitoring of the Sgr B2 cloud with $\mathrm{X}$-ray instruments shows flux variability on time scales of 10 years (Terrier et al. 2010). This variability can be naturally interpreted as a measure of the flare duration. Although the $\mathrm{X}$-ray luminosity and proton acceleration in Sgr A* should not necessarily correlate, it is interesting to explore proton flaring on decadal timescales.

In Figure 8, we compare the spectra of gamma-ray emission resulting from realization of three different scenarios: (1) a proton flare of 10 years duration that occurred 300 years ago, (2) a constant source that switched on $10^{4}$ years ago, and (3) a proton flare in addition to a constant source of protons-i.e., the superposition of (1) and (2). To fit the data, we took the size of the gamma-ray emission region to be $R=8$ pc, parameters of diffusion of $\beta=0.65, \kappa=1$, and an initial proton spectrum described by a power law of the form $Q(E) \propto E^{-2} \exp \left(-E / E_{\text {cut }}\right)$ (where $E_{\text {cut }}$ is the energy at which the spectrum cuts off and is analogous to the case described above where it was fixed at $100 \mathrm{TeV}$ by the HESS observations), and a proton injection rate of $1.9 \times 10^{39} \mathrm{erg} \mathrm{s}^{-1}$ for the constant source and total energy release in protons during the flare $W_{p} \simeq 6 \times 10^{50} \mathrm{erg}$. Note that as long as the duration of the flare is shorter than the time between observation and occurrence of the flare, the precise details of the history of the particle acceleration are immaterial. For a proton flare coinciding with the inferred X-ray flare of 10 years duration an average proton injection rate, $Q=W_{p} / \Delta t \simeq 1.9 \times 10^{42} \mathrm{erg} \mathrm{s}^{-1}$ would be implied and the ratio between the luminosity of the constant and flare state would then be comparable to the ratio derived in X-rays.

For the parameter set described above, the 300 year old flare cannot have a strong impact on the observed TeV spectrum, since 


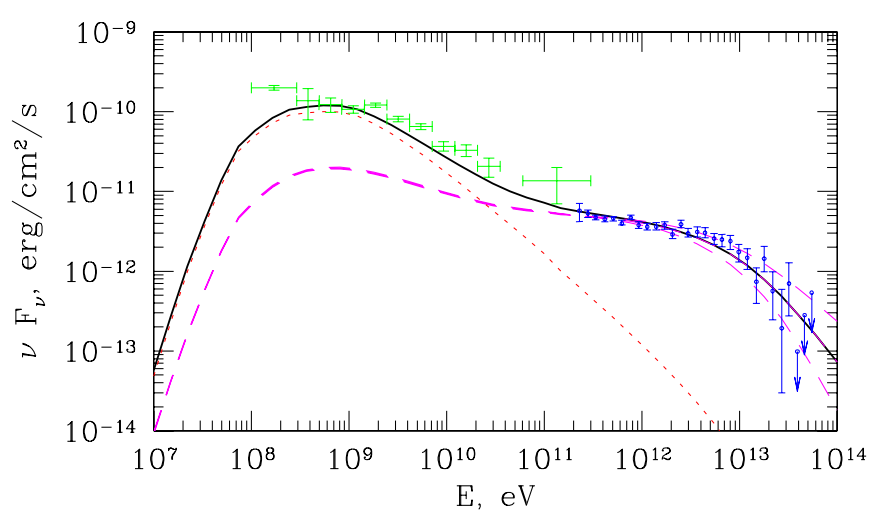

Figure 8. Combined Fermi (green points) and HESS (blue points) explained by the superposition (black solid line) of a proton flare of 10 years duration occurring 300 years ago (magenta dashed line) and a constant source that switched on $10^{4}$ years ago (red dotted line). See Section 4 for model parameters. The upper and lower dashed lines at high energies correspond to high-energy cutoff values of 100 and $50 \mathrm{TeV}$. The solid curve assumes $E_{\text {cut }}=75 \mathrm{TeV}$. Further higher energy data are needed before the range of this parameter can be further restricted.

(A color version of this figure is available in the online journal.)

most of the high-energy protons from the flare have already escaped. On the other hand, the emission at $\mathrm{GeV}$ energies is produced by protons from the flare which are still diffusively trapped in the gamma-ray production region. To explain the TeV data, we require much slower diffusion or a fresh injection of protons contributed by, for example, a very recent flare or by the continuous injection of protons. The case of superposition (solid line) of the flare (dashed line) and persistent (dotted line) components of protons is shown in Figure 8. For the chosen parameters, $\mathrm{GeV}$ gamma-rays are dominated by the flare component of protons, while $\mathrm{TeV}$ gamma-rays are mainly produced by protons from the persistent component.

We are thus able to reproduce the observed broadband spectrum of gamma-rays in different ways. The total energy required in relativistic protons currently trapped in the gammaray production region varies from $10^{49}$ to $10^{51} \mathrm{erg}$ for different models that can be injected in very different ways. In reality there has probably been a series of flares with different energetic signatures occurring throughout the lifetime of the central source.

Evidently the observed spectral and temporal properties of the $\mathrm{GC}$ at various wavebands are not enough to constrain all the parameters in our model. Additional information can be extracted, in principle, from the gamma-ray morphology of the inner arcminute region: above, we showed that different sets of parameters describing the observed spectral properties result in very different radial profiles (e.g., Figure 7). However, with the angular resolutions of the current space- and ground-based detectors, we cannot distinguish between the different radial profiles. Such information might be recovered by observations of synchrotron emission of secondary electrons from decays of charged pions, accompanying the production of gamma-rays from neutral pions. Since through this decay channel secondary electrons (and positrons; hereafter electrons only) and gamma-rays are produced concomitantly with similar energy distributions, we can directly connect the frequency of synchrotron photons from secondary electrons with the energy of the "genetically" connected gamma-rays:

$$
\epsilon \simeq 100\left(\frac{B}{10^{-4} \mathrm{G}}\right)\left(\frac{E_{\gamma}}{1 \mathrm{GeV}}\right)^{2} \mathrm{MHz},
$$

where the magnetic field is normalized to the probable minimum value expected in the region (Crocker et al. 2010).

Thus, to a first approximation, the morphology of the synchrotron radiation of "hadronic" origin should reflect the morphology of $\mathrm{GeV}$ gamma-rays. While at sub-GHz frequencies, GC radio photons are attenuated by free-free absorption in dense $\mathrm{H}$ II regions within and between the GC and the Earth (see Crocker et al. 2010), synchrotron emission at $\gtrsim 1 \mathrm{GHz}$ arrives without significant attenuation. The emissivity of synchrotron radiation of secondary electrons in the inner few pc of the Galaxy has been studied by Crocker et al. (2007), based on a model where the interactions of protons, diffusing away from an assumed central source, supply the observed, point-like TeV signal (Ballantyne et al. 2007). These authors then compared the predicted synchrotron emission to the $\mathrm{GHz}$ radio frequency spectrum, and found that essentially all the diffuse, non-thermal $\mathrm{GHz}$ radio emission from the central parsecs of the Galaxy could be explained as due to emission of secondary electrons (similar conclusions were obtained by Liu et al. 2006a). Therefore, we anticipate that the new Fermi data combined with available radio measurements should allow us to constrain significantly the parameter space of models positing that the $\mathrm{GeV}$ and $\mathrm{TeV}$ gamma-ray emission of the GC is due to hadronic interactions in the central few parsecs of GC. Analysis of the morphology of radio emission holds out particular promise here, but since the results of such an analysis are beyond the scope of this paper, they will be presented elsewhere.

It should be noted that CR electrons produce photons not only in the radio domain; their bremsstrahlung emission can be an important source of high-energy gamma-rays. Comparing the synchrotron and bremsstrahlung cooling times:

$$
\begin{aligned}
t_{\text {synch }} & \simeq 4 \times 10^{12}\left(\frac{B}{10^{-5} \mathrm{G}}\right)^{-2}\left(\frac{E_{e}}{1 \mathrm{TeV}}\right)^{-1} \mathrm{~s}, \\
t_{\text {bremms }} & \simeq 1.2 \times 10^{12}\left(\frac{n_{\mathrm{H}}}{1000 \mathrm{~cm}^{-3}}\right)^{-1} \mathrm{~s},
\end{aligned}
$$

we conclude that bremsstrahlung losses dominate over synchrotron at energies below:

$$
E_{e} \lesssim 30\left(\frac{n_{\mathrm{H}}}{10^{3} \mathrm{~cm}^{-3}}\right)\left(\frac{10^{-4} \mathrm{G}}{B}\right)^{2} \mathrm{GeV}
$$

This suggests that bremsstrahlung will dominate at energies less than $\sim 30 \mathrm{GeV}$ for fiducial $n_{\mathrm{H}}$ and $B$ values.

The relative importance of electron bremsstrahlung in producing the observed gamma-ray emission is characterized by the ratio of cooling times of electrons and protons associated with the bremsstrahlung and neutral pion production, respectively: $q_{\gamma}^{\mathrm{br}} / q_{\gamma}^{\pi^{0}} \simeq\left(3 t_{p p} / t_{\mathrm{br}}\right) f \simeq 4 f$, where $f=n_{e} / n_{p}$ is the electron-to-proton energy density ratio (Aharonian 2004). Hence, if the ratio of protons to electrons is $\gg 1$, then $\pi^{0}$-decay gamma-rays dominate over bremsstrahlung. The contribution of electron bremsstrahlung to $\mathrm{TeV}$ gamma-rays is quite limited because of the severe energy losses of VHE electrons due to the synchrotron and IC losses.

Finally, we note that the Fermi data presented here cannot be explained by the IC models proposed by Atoyan \& Dermer (2004) and Hinton \& Aharonian (2007). While these models are in a good agreement with HESS data, they predict that the energy flux in the GeV part of the spectrum should be smaller than in $\mathrm{TeV}$ range, apparently contrary to the Fermi observations. 


\section{SUMMARY}

We have analyzed 25 months of Fermi data on the GC region. The Fermi-LAT source 1FGL J1745.6-2900 lies within the error box of HESS source J1745-290. We found that, while below $5 \mathrm{GeV}$ the spectrum of 1FGL J1745.6-2900 has a photon spectral index similar to the HESS source, the spectrum at higher energies is better described by a steeper spectral index. We have formulated a model which produces a photon spectrum that can naturally explain the observed broadband gamma-ray emission. This model considers the hadronic interactions of relativistic protons which, having diffused away from a central source, presumably the central black hole, fill the inner few parsecs of our Galaxy. We have explored the parameter space of our model in terms of the geometry, characteristics of the diffusion coefficient, and injection rate history.

We have shown that the available spectral information can be well described with different sets of model parameters and that additional information is required to distinguish model scenarios. Such information can be obtained in principle from the spatial distribution of the observed gammaray emission; however, the required arcsecond resolution cannot be reached by current gamma-ray telescopes. On the other hand, synchrotron emission from the secondary electrons and positrons expected in our model might be detected by radio telescopes which possess an angular resolution high enough for the purposes of distinguishing between model parameters.

The authors thank Venya Berezinsky for discussions on the problems around the transition from the diffusive to the rectilinear transport regime and the anonymous referee for very useful comments. The authors also acknowledge the SFI/ HEA Irish Centre for High-End Computing (ICHEC) for the provision of computational facilities and support. The work of D.M. was supported by grant 07/RFP/PHYF761 from Science Foundation Ireland (SFI) under its Research Frontiers
Programme. During this work, R.M.C. has been the recipient of a Marie Curie Fellowship.

\section{REFERENCES}

Acero, F., et al. 2010, MNRAS, 402, 1877

Aharonian, F. A. 2004, Very High Energy Cosmic Gamma Radiation: A Crucial Window on the Extreme Universe (River Edge, NJ: World Scientific)

Aharonian, F., Buckley, J., Kifune, T., \& Sinnis, G. 2008, Rep. Prog. Phys., 71, 096901

Aharonian, F., \& Neronov, A. 2005a, ApJ, 619, 306

Aharonian, F., \& Neronov, A. 2005b, Ap\&SS, 300, 255

Aharonian, F., et al. 2004, A\&A, 425, L13

Aharonian, F., et al. 2006, Nature, 439, 695

Aharonian, F., et al. 2009, A\&A, 503, 817

Aloisio, R., Berezinsky, V., \& Gazizov, A. 2009, ApJ, 693, 1275

Atoyan, A., \& Dermer, C. D. 2004, ApJ, 617, L123

Atwood, W. B., et al. 2009, ApJ, 697, 1071

Ballantyne, D. R., Melia, F., Liu, S., \& Crocker, R. M. 2007, ApJ, 657, L13

Ballantyne, D. R., Schumann, M., \& Ford, B. 2010, MNRAS, in press (arXiv:1008.2661)

Cohen-Tanugi, J., Pohl, M., Tibolla, O., \& Nuss, E. 2009, in Proc. 31st ICRC (Lodz), 645 (http://icrc2009.uni.lodz.pl/proc/pdf/icrc0645.pdf)

Crocker, R. M., Jones, D., Ballantyne, D. R., \& Melia, F. 2007, ApJ, 664, L95

Crocker, R. M., Jones, D. I., Melia, F., Ott, J., \& Protheroe, R. J. 2010, Nature, 463, 65

Fryer, C. L., Liu, S., Rockefeller, G., Hungerford, A., \& Belanger, G. 2007, ApJ, 659,389

Ginzburg, V. L., \& Syrovatskii, S. I. 1964, The Origin of the Cosmic Rays (Oxford: Pergamon)

Hinton, J. A., \& Aharonian, F. A. 2007, ApJ, 657, 302

Koyama, K., Inui, T., Matsumoto, H., \& Tsuru, T. G. 2008, PASJ, 60, 201

Koyama, K., et al. 1996, PASJ, 48, 249

Law, C. J., Yusef-Zadeh, F., Cotton, W. D., \& Maddalena, R. J. 2008, ApJS, 177,255

Liu, S., Melia, F., Petrosian, V., \& Fatuzzo, M. 2006a, ApJ, 647, 1099

Liu, S., Petrosian, V., Melia, F., \& Fryer, C. L. 2006b, ApJ, 648, 1020

Rando, R., et al. 2009, arXiv:0907.0626

Revnivtsev, M. G., et al. 2004, A\&A, 425, L49

Sunyaev, R. A., Markevitch, M., \& Pavlinsky, M. 1993, ApJ, 407, 606

Syrovatskii, S. I. 1959, Astron. Zh., 36, 17

Terrier, R., et al. 2010, ApJ, 719, 143

Vitale, V., \& Morselli, A. 2009, arXiv:0912.3828

Wang, Q. D., Lu, F. J., \& Gotthelf, E. V. 2006, MNRAS, 367, 937 